\title{
4-NONILFENOL: EFECTOS, CUANTIFICACIÓN Y MÉTODOS DE REMOCIÓN EN AGUAS SUPERFICIALES Y POTABLES
}

\author{
4-NONYLPHENOL: EFFECTS, QUANTIFICATION AND METHODS \\ OF REMOVAL IN SUPERFICIAL AND DRINKING WATER
}

\author{
${ }^{1}$ Gloria Maria Doria Herrera \\ ${ }^{2}$ Gustavo Peñuela Meza \\ ${ }^{3}$ Gloria Cristina Valencia \\ ${ }^{1}$ Universidad de Antioquia, ${ }^{2}$ Universidad de Antioquia, ${ }^{3}$ Universidad Nacional de colombia- sede Medellin \\ ${ }^{1}$ gloria.doria@udea.edu.co, ${ }^{2}$ gustavo.penuela@udea.edu.co \\ ${ }^{3}$ gcvalenceunal.edu.co
}

\section{RESUMEN}

Los contaminantes emergentes constituyen un grupo de sustancias cuya regulación está siendo abordada, debido al incremento de su presencia en cuerpos de agua asociado principalmente a las acciones antropogénicas. Estos compuestos, que son caracterizados como disruptores endocrinos a bajas concentraciones, causan daños irreversibles en los ecosistemas y están siendo objeto de estudio especialmente en las últimas dos décadas. Para mitigar su impacto y presencia en los ecosistemas, se han estudiado alternativas para lograr su remoción, basadas en procesos de adsorción, electrocoagulación, filtración por membrana y procesos de oxidación avanzada (AOP). Específicamente el contaminante emergente 4-nonilfenol (4-NF), un compuesto perteneciente a la familia de los alquilfenoles, es utilizado como principio activo de tensoactivos no iónicos para uso en detergentes, dispersantes, emulsionantes y solubilizantes, estando presente entre otros, en formulaciones producto de aseo, de plaguicidas y de pinturas, siendo su principal vía de acceso al ambiente a través de aguas residuales. Esta revisión bibliográfica reúne información acerca de algunas propiedades fisicoquímicas del 4-NF, toxicidad, métodos de cuantificación y remoción.

Palabras clave: toxicidad, contaminante, 4-nonil fenol, análisis, cuantificación.

\section{ABSTRACT}

The emergent contaminants are a group of non-regulated substances with low concentrations that cause irreversible damage to ecosystems and have been mostly identified as endocrine disruptors, so have gained great interest in the last 20 years. To mitigate its impact and presence in ecosystems, alternatives have been proposed to achieve their removal, such as adsorption, electrocoagulation, 
membrane filtration and advanced oxidation processes (AOP). 4- nonylphenol (4-NF) is used as a precursor in the manufacture of nonionic surfactants for use in detergents, dispersing, emulsifying, pesticide formulations, paints and personal care products, so its main access road to the environment it is by sewage, however, has become increasingly important environmental level, by various effects generated when entering aquatic ecosystems and the variety of isomers which can be found in the environment. In this review information about some physicochemical properties of 4-NF, toxicity, quantification method considering analytical and instrumental conditions, and methods of removal is collected.

Keywords: toxicity, pollutant, 4-nonylphenol, emerging, quantification

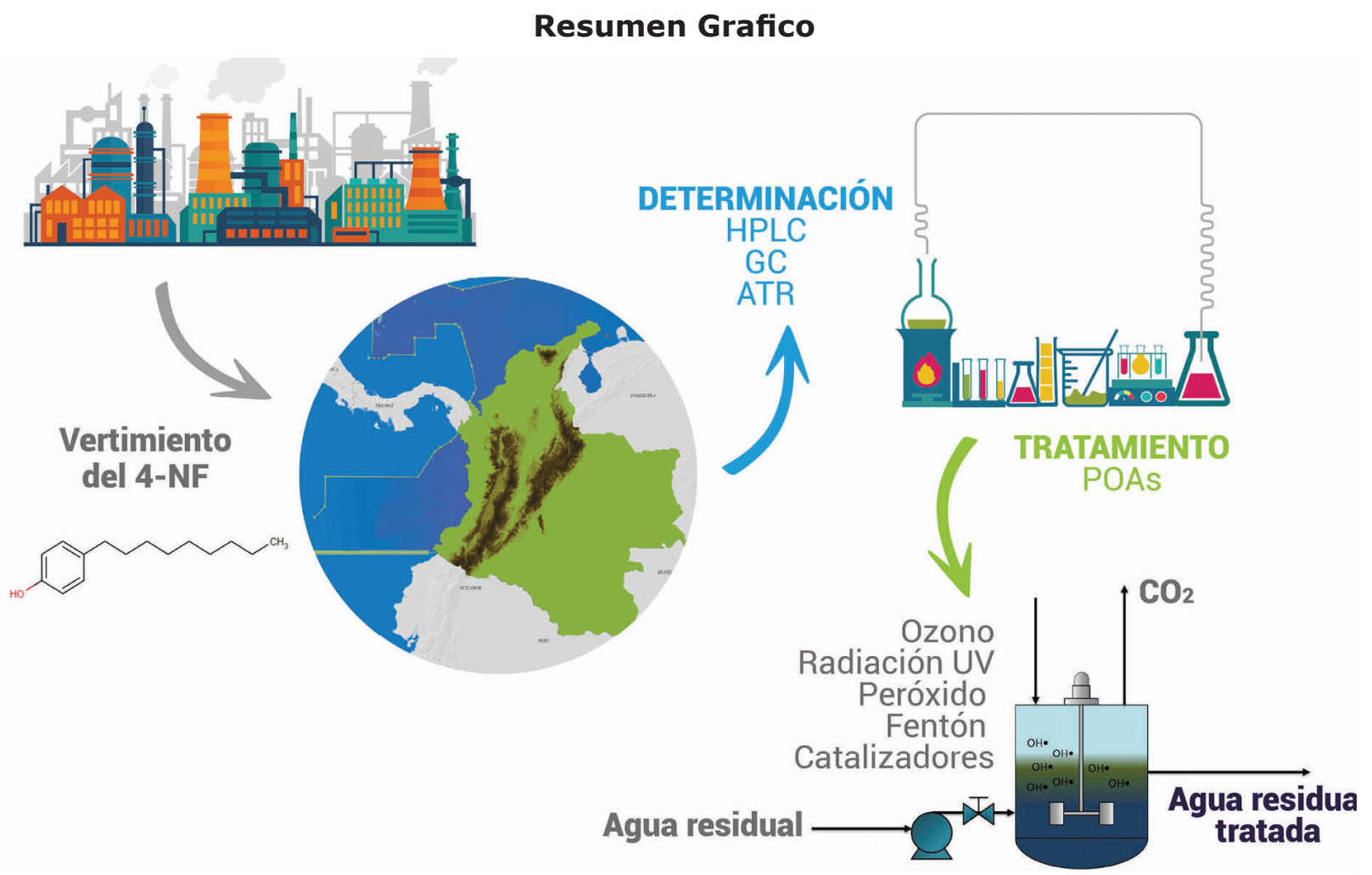

\section{INTRODUCCIÓN}

Los contaminantes emergentes son compuestos que previamente no han sido reconocidos como tal y que, a pesar de encontrarse en bajas concentraciones en ambientes acuáticos y terrestres, tienen el potencial de causar efectos adversos sobre la salud y el medio ambiente (EPA, 2015), en especial cuando forman compuestos secundarios con capacidad recalcitrante y habilidad de biomagnificarse. Es preocupante y se constituye como un problema de salud pública que estos contaminantes no sean removidos en las plantas de tratamiento de aguas residuales ni en aguas de consumo humano, pues debido a la escasa regulación al respecto, ni siquiera son monitoreados y mucho menos se aplican metodologías que sean eficientes para su tratamiento. Esto viene motivando diversas investigaciones, especialmente apoyadas con el desarrollo de métodos analíticos más sensibles y avances a nivel 
instrumental, con los que se logra la detección de estos materiales en muestras ambientales incluso a niveles de $\mu \mathrm{g} / \mathrm{L}$ (Noguera-Oviedo \& Aga 2016). No obstante, aún se emplean metodologías que permiten concentrar la muestra para que pueda ser cuantificada.

Diversas son las fuentes de estos contaminantes e incluyen su presencia en formulaciones de productos químicos farmacéuticos y de higiene personal (PPCPs, por su sigla en inglés: pharmaceuticals and personal care products), detergentes para el hogar y la industria, pesticidas, plastificantes, metabolitos de transformación, síntesis de nuevos materiales, drogas de abuso, aditivos en gasolina y retardantes de fuego, y de forma general, diversos compuestos de origen doméstico e industrial (Gil et al. 2012; Lin et al., 2016). En la Figura 1 (ver anexo) se reúnen reportes referentes al análisis de contaminantes emergentes, así como las innovaciones e instrumentación. La información registrada en esta figura de 1950 al 2005 fue compilada por Noguera-Oviedo \& Aga (2016), y ha sido complementada con otras investigaciones relevantes hasta el 2017 como objeto de la presente revisión.

El este artículo se presenta una revisión bibliográfica del 4-nonilfenol (4-NF) que incluye toxicidad, métodos de cuantificación y remoción. Especialmente se hace referencia a investigaciones relacionadas con la aplicación de tecnologías que permiten mitigar la presencia de microcontaminantes en aguas, destacando la aplicación de procesos de oxidación avanzada por el interés de lograr la mineralización de estos compuestos.

\section{ANTECEDENTES}

\section{Propiedades fisicoquímicas}

El 4-NF de la familia de los alquilfenoles es utilizado principalmente como precursor en la fabricación de tensoactivos no iónicos para uso en detergentes, desengrasantes, dispersantes, emulsionantes, estabilizantes, formulaciones de plaguicidas, pinturas y productos para el cuidado personal (Gil et al. 2012; Martínez-Zapata et al., 2013; Lin et al., 2016; Diehl et al., 2005). Su capacidad como tensoactivo le permite modificar la tensión superficial de mezclas y en consecuencia la permeabilidad de materiales y tejidos sobre los que se aplica. En su estructura (Figura 2), se reconoce al anillo fenólico como la parte polar, por lo que capacidad de solubilizarse en agua se verá afectada en función del tamaño de la cadena carbonada que corresponde al segmento apolar cuyo efecto inductivo disminuye al aumentar la longitud de la cadena. Al aumentar la longitud de la cadena alquílica en la molécula (menos ramificaciones), disminuye su solubilidad en agua e incrementa su solubilidad en presencia de otros solventes de carácter orgánico y con ello su peligrosidad. Por el contrario, si se hace más corta la cadena lateral, mejoraría la solubilidad en medios acuosos, aumentando su persistencia (Ying et al., 2002).

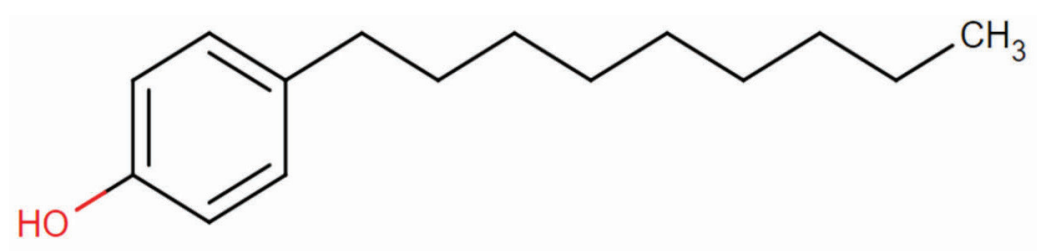

Figura 2. Estructura química del 4-n-Nonilfenol.Fuente los autores 
El 4-NF representa alrededor del $90 \%$ de los nonilfenoles, del cual existen teóricamente 211 isómeros constitucionales y si se consideran los estereoisómeros, este número incrementa a 550. Estos isómeros presentan diferente estrogenicidad y biodegradabilidad (Lu \& Gan 2014). En la Figura 3 se muestran algunos compuestos de la familia de los 4-NF encontrados en una muestra comercial.
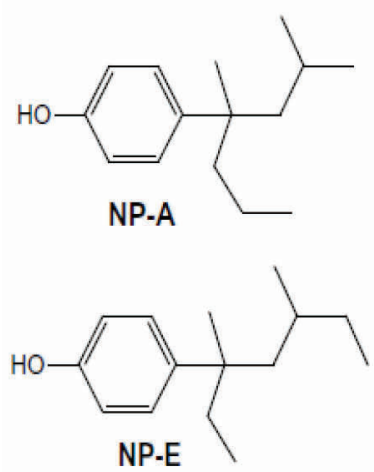

NP.E

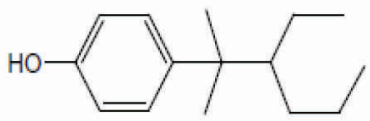

NP-I

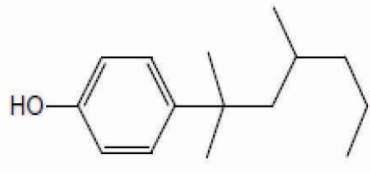

NP-B

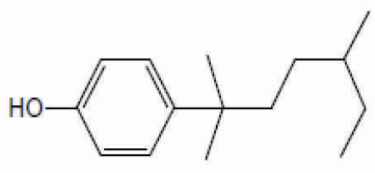

NP-F

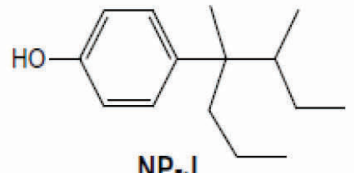

NP.J

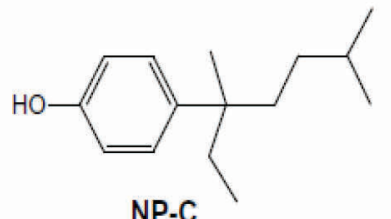

NP-C

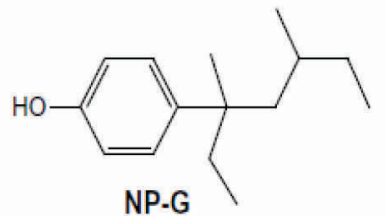

Diastereoisomero de NP-E

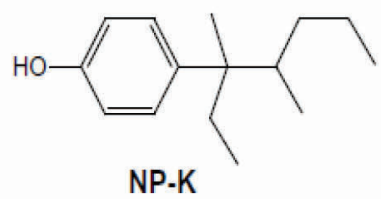

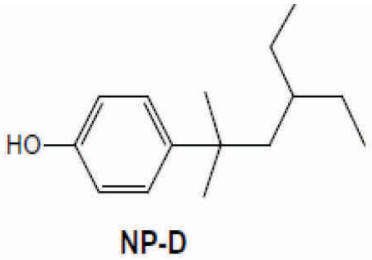
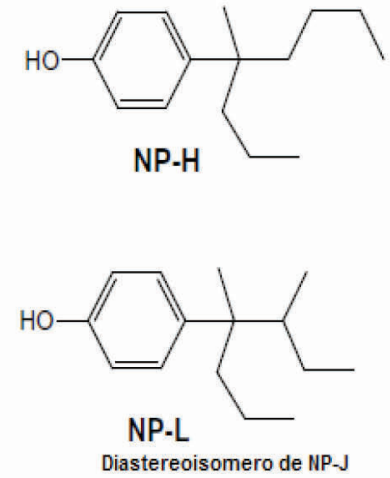

Figura 3. Estructuras químicas de isómeros del 4-NF presentes en una muestra comercial.

Tomado de Uchiyama et. al. (2008).

El 4-NF se produce industrialmente por la alquilación de fenol con nonano por catálisis en condiciones de ácidas (Soares et al., 2008). diversos estudios han reportado más de 100 isómeros de la familia del 4-NF y el producto comercial en condiciones ambientales es un líquido viscoso translucido entre transparente y ligeramente amarillento e inmiscible con agua (Ieda \& Horii, 2005; Pontolillo et al., 2009).

\section{Fuente de emisión}

En la Figura 4, se ilustra cómo el 4-NF es incorporado al recurso hídrico y superficies terrestres provenientes de actividades domésticas e industriales. Debido a sus propiedades fisicoquímicas entre las que incluye ser altamente hidrofóbico, tienden a adsorberse en sólidos suspendidos y, en consecuencia, es factible encontrarlo en sedimentos. Sin embargo, se ha detectado su presencia en aguas superficiales en concentraciones entre $3 \times 10^{-4}$ y $37,3 \mathrm{mg} / \mathrm{m}^{3}$ (Careghini et al., 2014), posiblemente debido al aporte de las descargas de aguas residuales de zonas urbanas. Ya en los sistemas hídricos, el 4-NF es capaz de biomagnificarse y llegar al ser humano. 


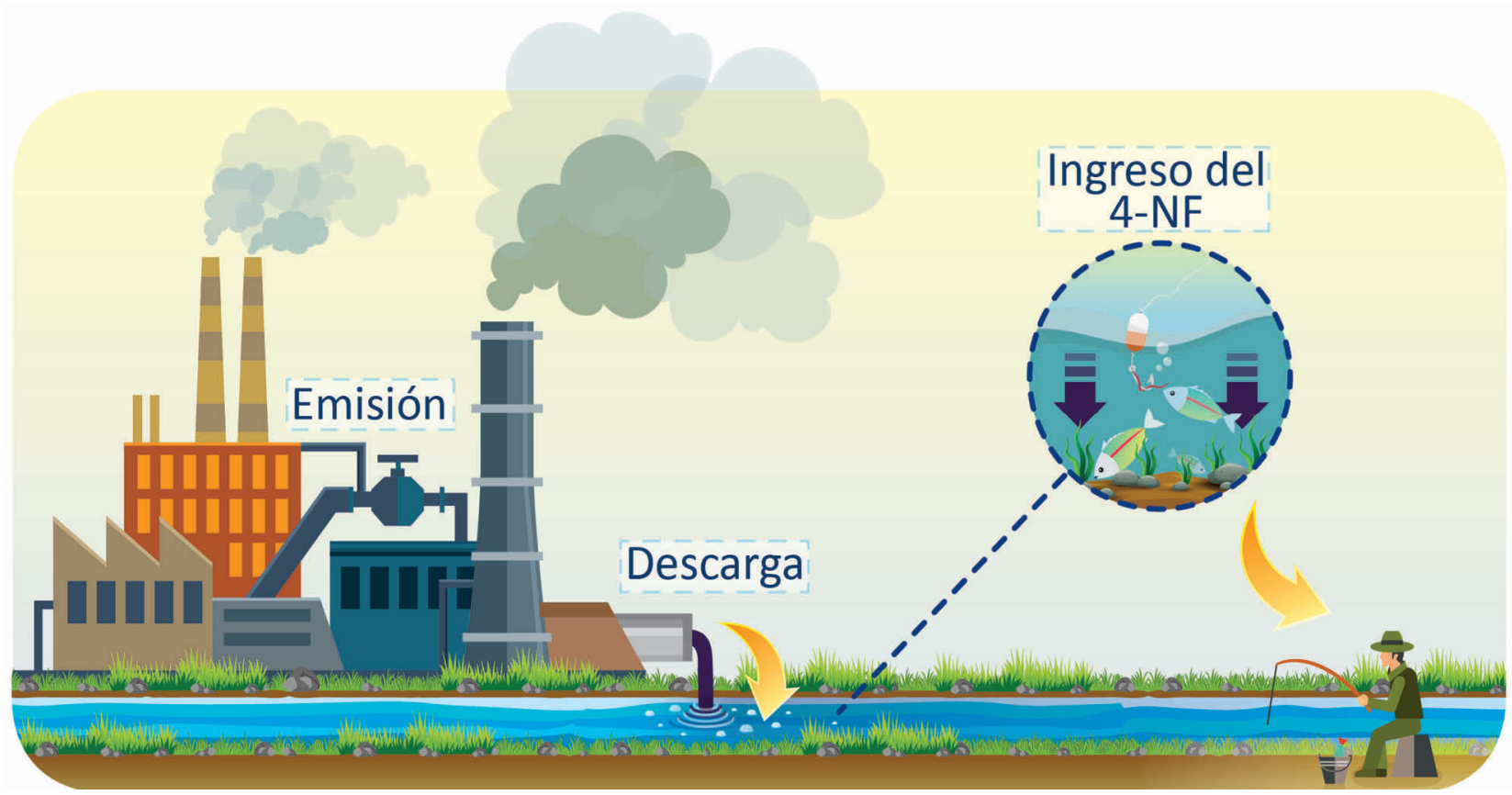

Figura 4. Descarga del 4-nonilfenol en los cuerpos de agua e ingreso al ser humano. Fuente los autores

En la Tabla 1 se muestran algunos estudios que incluyen la detección del 4-NF en aguas (Bolong et al., 2009; Lin, Yu, \& Chen 2016). Gracias a los reportes originados de estas y otras investigaciones, se espera contribuir en la sensibilización de la comunidad, referente a la responsabilidad inherente al uso de productos que contengan este y otros contaminantes emergentes, y su adecuada disposición final, así como motivar a quienes intervienen en los sistemas de regulación, para que se promueva el control de las descargas de este compuesto.

Tabla 1. Hallazgos del 4-NF y otros contaminantes emergentes en aguas naturales, superficiales y potables.

\begin{tabular}{|c|c|c|c|c|c|}
\hline Tipo De Aguas & Sitio & $\begin{array}{l}\text { Compuesto } \\
\text { Detectado }\end{array}$ & $\begin{array}{c}\text { Técnica } \\
\text { implementada }\end{array}$ & Concentración & Referencia \\
\hline $\begin{array}{l}\text { Aguas residuales } \\
\text { industriales }\end{array}$ & $\begin{array}{l}\text { Bélgica } \\
\text { e Italia }\end{array}$ & Nonilfenol & $\begin{array}{l}(\mathrm{RP}-\mathrm{LC}) \\
(\mathrm{ESI}),(\mathrm{MS}) \text { y }(\mathrm{API})\end{array}$ & Max. $2.5 \mathrm{mg} / \mathrm{L}$ & $\begin{array}{l}\text { Loos et al., } \\
\text { (2007) }\end{array}$ \\
\hline Consumo & Alemania & $\begin{array}{l}\text { Nonilfenol } \\
\text { Estrógeno } \\
\text { Estradiol }\end{array}$ & $\begin{array}{l}\text { GC-MS usando método } \\
\text { HPLC y se realiza } \\
\text { extracción CL }\end{array}$ & $\begin{array}{l}8.0(\mathrm{ng} / \mathrm{L}) \\
0.4(\mathrm{ng} / \mathrm{L}) \\
0.3(\mathrm{ng} / \mathrm{L})\end{array}$ & $\begin{array}{l}\text { Kuch \& } \\
\text { Ballschmiter, } \\
(2001)\end{array}$ \\
\hline $\begin{array}{l}\text { Residuales } \\
\text { Municipal }\end{array}$ & UK & $\begin{array}{l}\text { Nonilfenol } \\
\text { Estrógenos } \\
\text { Estradiol }\end{array}$ & $\begin{array}{l}\text { GC-MS/ uso como } \\
\text { método de extracción y } \\
\text { purificación HPLC }\end{array}$ & $\begin{array}{l}1.2-1.7 \mathrm{ng} / \mathrm{L} \\
15-220 \mathrm{ng} / \mathrm{L} \\
7-88 \mathrm{ng} / \mathrm{L}\end{array}$ & $\begin{array}{l}\text { Rodgers- } \\
\text { gray et al. } \\
(2001)\end{array}$ \\
\hline Aguas superficiales & Alemania & $\begin{array}{l}\text { Nonilfenol } \\
\text { Estrógenos } \\
\text { Estradiol } \\
\text { Etinilestradiol }\end{array}$ & GC-MS & $\begin{array}{l}199 \mathrm{ng} / \mathrm{L} \\
3.4 \mathrm{ng} / \mathrm{L} \\
0.9 \mathrm{ng} / \mathrm{L} \\
1.4 \mathrm{ng} / \mathrm{L}\end{array}$ & $\begin{array}{l}\text { Kuch \& } \\
\text { Ballschmiter, } \\
(2001)\end{array}$ \\
\hline
\end{tabular}




\begin{tabular}{|c|c|c|c|c|c|}
\hline Tipo De Aguas & Sitio & $\begin{array}{l}\text { Compuesto } \\
\text { Detectado }\end{array}$ & $\begin{array}{c}\text { Técnica } \\
\text { implementada }\end{array}$ & Concentración & Referencia \\
\hline Aguas superficiales & Japón & $\begin{array}{l}\text { Estrógenos } \\
\text { Estradiol }\end{array}$ & \multirow[b]{2}{*}{ GC-MS } & $\begin{array}{l}80-1240 \mathrm{ng} / \mathrm{L} \\
2.7-48 \mathrm{ng} / \mathrm{L}\end{array}$ & \multirow{2}{*}{$\begin{array}{l}\text { Kuch \& } \\
\text { Ballschmiter, } \\
(2001)\end{array}$} \\
\hline \multirow[t]{2}{*}{ Aguas superficiales } & Alemania & $\begin{array}{l}\text { Nonilfenol } \\
\text { Estrógenos } \\
\text { Estradiol }\end{array}$ & & $\begin{array}{l}34 \mathrm{ng} / \mathrm{L} \\
0.7 \mathrm{ng} / \mathrm{L} \\
0.6 \mathrm{ng} / \mathrm{L}\end{array}$ & \\
\hline & Japón & $\begin{array}{l}\text { Nonilfenol } \\
\text { Estradiol }\end{array}$ & GC & $\begin{array}{l}250 \mathrm{ng} / \mathrm{L} \\
2.1 \mathrm{ng} / \mathrm{L}\end{array}$ & $\begin{array}{l}\text { Bolong et al., } \\
(2009)\end{array}$ \\
\hline Agua de mar & $\begin{array}{l}\text { Norte } \\
\text { América }\end{array}$ & $\begin{array}{l}\text { Nonilfenol } \\
\text { Etoxilatos del } \\
\text { Nonilfenol }\end{array}$ & GC & $0.42 \mathrm{mg} / \mathrm{L}$ & $\begin{array}{l}\text { Diehl et al., } \\
(2012)\end{array}$ \\
\hline $\begin{array}{l}\text { Aguas superficiales } \\
\text { (16 Ríos) }\end{array}$ & Taiwán & $\begin{array}{l}\text { Nonilfenol } \\
\text { Bisfenol A }\end{array}$ & Cromatografía liquida & $\begin{array}{l}0.02-3.94 \mathrm{mg} / \mathrm{L} \\
0.01-44.65 \mathrm{mg} / \mathrm{L}\end{array}$ & $\begin{array}{l}\text { C. C. Lee et } \\
\text { al., (2013) }\end{array}$ \\
\hline Agua de mar & Japón & Nonilfenol & $\begin{array}{l}\text { HPLC/ Detector de } \\
\text { fluorescencia }\end{array}$ & $5-1000 \mathrm{ng} / \mathrm{mL}$ & $\begin{array}{l}\text { Cherniaev et } \\
\text { al. (2016) }\end{array}$ \\
\hline Aguas superficiales & $\begin{array}{l}\text { Estados } \\
\text { Unidos }\end{array}$ & $\begin{array}{l}\text { Nonilfenol } \\
\text { Otros } 67 \\
\text { contaminantes } \\
\text { emergentes } \\
\text { farmacéuticos }\end{array}$ & $\mathrm{HPLC} / \mathrm{MS}$ & $10^{3}-10^{4} \mathrm{ng} / \mathrm{L}$ & $\begin{array}{l}\text { Fairbairn et } \\
\text { al. (2016) }\end{array}$ \\
\hline Aguas de estuarios & $\begin{array}{l}\text { Estados } \\
\text { Unidos }\end{array}$ & $\begin{array}{l}150 \\
\text { compuestos } \\
\text { cuidado } \\
\text { personal, } \\
\text { farmacéuticos } \\
\text { (Nonilfenol) }\end{array}$ & LC & $506-1690 \mathrm{ng} / \mathrm{L}$ & $\begin{array}{l}\text { Meador et } \\
\text { al., (2016) }\end{array}$ \\
\hline
\end{tabular}

Fuente: los autores

\section{Toxicidad del 4-nolnilfenol}

El 4-NF se origina a partir de la degradación de etoxilatos de nonilfenol, compuestos ampliamente utilizados como tensoactivos industriales (Kuzikova, et al., 2016; Rabouan et al., 2012; Deborde, et al., 2008; Cheng et al., 2015; Verderame et al., 2011). Incluso el 4-NF es más tóxico que los mismos compuestos etoxilatos (Ying, 2002) y presenta riesgos por ser un disruptor endocrino, evidenciado este efecto en fauna acuática (Sumpter \& Jobling 1995). Se han reportado efectos estrogénicos por presencia de estos compuestos del 4-NF en cultivos de plantas como Atriplex hortensis L., Beta vulgaris var. altissima L. Cv. Bush,
Mono Leaves, Beta vulgaris var. esculenta L. cv. Monogold, Chenopodium quinoa L., Chenopodium rubrum L., Daucusmcarota L. CV. Nantaise, Glycine max L. Merr. cv. Mandarin, Hordeum vulgare L., Lycopersicon esculentum Mill., Triticum aestivum L. cv. Heines Koga II. y en vegetales: Esto puede presentarse por la adsorción en suelos, tras la formación de complejos (Bokern \& Harms, 1997; Zgoła et al., 2009; (Sayed et al.,2016).

Por otro lado, se pudo verificar la presencia de 4-NF en 60 productos alimenticios de supermercados alemanes, en concentraciones desde 0,1 a $19,4 \mu \mathrm{g} / \mathrm{kg}$ (peso fresco) y esto 
correlacionado con la tasa de consumo promedio reportado por los entes gubernamentales alemanes, asocian a posibles efectos secundarios adversos para la salud humana de alimentos contaminados por 4-NF (Guenther et al., 2002). Un estudio en aguas dulces, marinas y crustáceos, arrojan valores para la dosis letal media $\left(\mathrm{LC}_{50}\right.$ ) menores a 50 ppb (Vitali et al., 2004) por lo que es posible concluir que tras la exposición a concentraciones de orden $0,1 \mathrm{ppb}$ del 4-NF se incurre en alta peligrosidad para los seres humanos.

Por sus riesgos, ya en la Unión Europea (UE) y Canadá, han iniciado el tema de control de su uso en los procesos de manufactura, apoyados en resultados demostrados en investigaciones (Sumpter \& Jobling 1995). Para 2008, la Unión Europea (UE) presentó las Normas de Calidad Ambiental (NCA), fijando en 2,0 $\mathrm{\mu g} / \mathrm{l}$ la concentración máxima permitida en aguas superficiales continentales. En este se plantea el compromiso para el monitoreo, registro y los planes de regulación, por parte de los estados miembros (Greenpeace, 2010). Como fecha de referencia, los planes de seguimiento deberán ser presentados en septiembre del 2018, por lo que se percibe un proceso muy laxo en la emisión de normas y controles de este y otros contaminantes emergentes.

Las implicaciones del 4-NF, así como otros disruptores endocrinos, se enmarcan en el efecto que presentan sobre el sistema hormonal mediante la imitación de hormonas naturales, bloqueando su producción y/o inhibiendo su función, y en consecuencia afectando los procesos fisiológicos controlados a través de las hormonas. Particularmente, el 4-NF es un imitador de la hormona natural $17 \beta$-estradiol, compitiendo por el sitio de unión del receptor de estrógenos (Schirmer et al., 2006; Soares et al., 2008). Resultados obtenidos en estudios de su presencia en aguas residuales, superficiales y en ríos de países tales como Japón, Alemania, Italia y países bajos, en los que se han encontrado concentraciones hasta de 27 ng/L (Chen et al., 2014). Aunque se han implementado metodologías de remediación de tipo biológico, aun no es posible determinar el comportamiento ambiental y el destino de estos esteroides hormonales en diferentes ecosistemas (Ying et al., 2002).

La presencia de 4-NF ha sido evidenciada en ecosistemas acuáticos, reporto investigaciones en el lago Taihu en China con concentraciones en aguas de $116 \mu \mathrm{g} / \mathrm{L}$, mientras que el contenido de NP en la carne de camarón, hígado de pescado, pescado la grasa, la carne de pescado y la carne de mariscos fueron 108, 375,301 respectivamente, superando las cantidades determinadas en aguas, en el caso de las algas, tal es el caso del alga Cyclotella caspia, considerada un biomarcador de contaminación, se encontró que la EC50 fue de 0.18 ppm, dentro de los aspectos que se analizan en la inhibición son: ampliación del volumen, inclusión citoplásmica, agregación, clorofila empañada y ruptura celular (Liu, Dai, \& Wei, 2013).

Además, de la capacidad de bioacumulación del NF genera en los peces puede afecciones en la síntesis de la proteína asociada a la vitelogenina (VTG), generando un descontrol multihormonal que causa la feminización de peces machos, incluso a concentraciones de $1 \mu \mathrm{g} / \mathrm{L}$ (Sumpter \& Jobling, 1995). También se han encontrado lesiones histopatológicas en truchas marrones, con daños en branquias y tejido del intestino cuando son sometidas a concentraciones de 1 a 100 ppb (Shirdel \& Kalbassi, 2016). En mamíferos tales como ratones macho expuestos a concentraciones de $60 \mathrm{mg} / \mathrm{kg}$ se evidenció efectos adversos asociados con deficiencias hormonales, trastornos de metabolismo de fructosa y daño oxidativo testicular (Duan et al., 2016). 
La interacción del 4-NF en organismos vivos conduce de forma general a cambios en el sistema endocrino, generando problemas para la preservación de las especies. Específicamente para el hombre, cuyo ingreso de este compuesto al organismo puede darse por vía cutánea, ingestión o inhalación, tiene un efecto directo en el aborto y mutaciones a concentraciones de 0.41 $\pm 0.49 \mathrm{ng} / \mathrm{mL}$ (Peng et al., 2016; Wang et al., 2015). Lo anterior, permite determinar que el 4-NF genera afecciones tanto a nivel biológico como ambiental, concluyendo que la interacción del 4-NF con los ecosistemas es altamente nociva, en especial dada su capacidad de biomagnificarse.

\section{Técnicas de cuantificación}

En la identificación y cuantificación del 4-NF y sus isómeros, se han utilizado cotidianamente técnicas como la cromatografía líquida de alta resolución (HPLC) (She et al., 2012) y cromatografía de gases (GC), y en algunos casos el método de ELISA (Lu \& Gan, 2014; Vincent \& Sneddon, 2009; Céspedes et al., 2006). A pesar de que en GC se presenta buena sensibilidad en términos de cuantificación, hay algunas limitaciones en su aplicación asociadas con la volatilidad de los compuestos de interés, debido a que solo podrían separarse compuestos que puedan evaporarse a la temperatura máxima de trabajo en el horno. Sin embargo, aun cuando pueden utilizarse procesos de derivatización para reducir la temperatura de ebullición de los analitos, con HPLC se evitan estas limitaciones, siendo una metodología bandera en la determinación de este tipo de contaminantes, ampliando el espectro de posibilidades en el análisis, al poder modificar fase móvil (solventes), fase estacionaria y condiciones de elución.

Existen otros estudios que han demostrado la posibilidad de análisis del 4-NF por Espectroscopia Infrarroja con reflectancia atenuada
(ATR-FTIR) y análisis por RAMAN, sin embargo, las concentraciones de análisis estaban en el rango de 0 a $100 \mathrm{ppm}$, superando las concentraciones de trabajo para ser considerado un contaminante emergente (Duan et al., 2019). Así mismo, (Su, et al., 2017)estudios han reportado el uso de la técnica voltametríca para el análisis del 4-NF, reportando curvas de calibración en el rango $0.01 \mu \mathrm{M}-10 \mu \mathrm{M}$, por lo que reafirma que las técnicas más sensibles de análisis con las cromatográficas.

\section{Preparación de muestra para el análisis del 4-Nonilfenol}

Basados en las condiciones y características de la matriz donde se encuentre el compuesto a separar, así como la posible presencia de isómeros, puede ser útil la implementación de pretratamientos como se ilustran en la Tabla 2 (ver anexo), logrando mejores condiciones de separación. Estos pretratamientos incluyen extracción en fase sólida (SFE), extracciones líquido-líquido y complejación (Guerreiro et al., 2008). En matrices complejas, incluyendo los materiales biológicos, es imperativa la aplicación de métodos de extracción previa para luego proceder a la separación y cuantificación del contaminante, debido a que, en bajas concentraciones, las matrices complejas pueden enmascarar la presencia del analito, actuando incluso como "atrapadores" del compuesto de interés. La extracción en fase sólida en una de las metodologías más destacadas por ser de fácil manejo, debido a que los cartuchos vienen listos para ser utilizadas, y solo se requiere la solubilización de los componentes de la mezcla.

\section{Condiciones de operación para el análisis del 4-Nonilfenol}

Debido a la existencia de un gran número de isómeros del 4-NF y a que las muestras donde está presente este compuesto, sus isómeros, metabolitos y productos de degradación, 
en general son muy complejas, se requieren de una combinación estratégica de técnicas analíticas, que incluyen diversos los protocolos de extracción, así como diversas condiciones de operación para el desarrollo de métodos en la cuantificación de este tipo de analitos. La cromatografía es la técnica por excelencia en cuanto a la separación e identificación, existen estudios recientes que compilan métodos de preparación, toxicidad, legislación y determinación usando la cromatografía como la técnica más versátil para la cuantificación del 4-NF (Araujo, Bauerfeldt, \& Cid, 2017), sin embargo, estos reportan a grandes rasgos acerca de cada uno de los aspectos anteriormente mencionados, además incluyen los dos tipos de cromatografía como son gases y liquida, en esta investigación nos enfocamos en el HPLC y se da de forma detallada la identificación y cuantificación, así como para el seguimiento de sus procesos de degradación, se usan diversidad de columnas para HPLC, fases móviles, especificaciones instrumentales asociadas al tipo de detector y condiciones del equipo, que integran el trabajo con diferentes matrices donde se pueden encontrar compuestos domo el 4-NF y sus derivados, tal como se muestra en la Tabla 3 (ver anexo).

Se resalta el uso de UHPLC con detectores de fluorescencia, con sensibilidad entre 20 a 30 veces mayor al detector de arreglo de diodos (DAD). Lo anterior, aprovechando el hecho de ser compuestos fluoroforos. Para las señales de fragmentación destacadas se evidencia el pico del ion base m/z: 219. Los subproductos formados dependerán del tipo de tratamiento utilizado y si el caso de la aplicación de procesos de oxidación avanzada, también pueden generarse diversidad de productos, dadas las diferentes condiciones de reacción y el tipo de especie oxidante.

\section{Métodos de remoción del 4-Nonilfenol (4-NF)}

Se han usado diversos métodos de remoción tal como adsorción, filtración por membrana, osmosis inversa, nanofiltración, oxidación química entre otros (Villegas et al., 2016). Específicamente para el 4-NF se han propuesto diversas metodologías de remoción dentro de los que se destaca la electroadsorción por nanotubos de carbono ( $\mathrm{Li}$ et al., 2014), sin embargo, los riesgos asociados a la adsorción tales como cambios en el $\mathrm{pH}$, temperatura, concentración inicial se vuelven factores determinantes en la eficiencia de adsorción, por ello se ha potenciado la aplicación de los procesos de oxidación avanzada, los cuales tienen la presencia del radical libre hidroxilo, poderoso agente oxidante, que puede conducir a la mineralización de los contaminantes.

Dentro del grupo de los procesos de oxidación avanzada se destaca Fenton, Fotofenton, Fenton asistido, sonoquímica, electroquímica, fotocatálisis, ozonación, $\mathrm{UV} / \mathrm{H}_{2} \mathrm{O}_{2}$, sin embargo, en esta revisión nos dedicamos solo a investigaciones reportadas para el tratamiento del 4-NF en aguas. Dentro de los estudios para la degradación para el 4-NF usando los procesos de oxidación avanzada como $\mathrm{UV} / \mathrm{H}_{2} \mathrm{O}_{2}$, Fenton y Fotofenton, lográndose los mejores resultados a $\mathrm{pH}$ básicos con $\mathrm{UV} / \mathrm{H}_{2} \mathrm{O}_{2}$, sin embargo, el escalamiento no ha sido satisfactorio (Dulov et al., 2013; Peng et al., 2016) reportaron la degradación del 4-NF usando luz solar simulada y el efecto de los nitratos, el ion férrico y el ion radical carbonato en el proceso fotodegradativo, alcanzándose porcentajes de degradación satisfactorias (98\%).

Se han reportado investigaciones con agua residuales de las industrias textileras y de curtiembres conteniendo 4-NF, alcanzándose porcentajes de degradación de $42 \%$ (Pothitou \& 
Voutsa, 2008). En otro estudio se reportó que el 4-NF alcanzó porcentajes de degradación de aproximadamente 60\% (Nakada et al., 2006).

Xu et al. (2016) realizaron la degradación del 4-NF usando la sonoquímica, el cual no requiere aditivos químicos y genera menos subproductos peligrosos. En este estudio se evidenció, que el radical libre hidroxilo fue la principal vía de oxidación del 4-NF. No se comprobó la mineralización, pero se identificaron varios intermediarios de reacción. (Dzinun et al. 2016) usando la fotocatálisis heterogénea con $\mathrm{TiO}_{2}$, mediante una membrana de fibra hueca recubierta con doble capa con dióxido de titanio inmovilizada lograron la degradación del 4-NF.

Bechambi et al. (2016) usaron wurtzita dopada con $\mathrm{Ag}(\mathrm{I})$ como material fotocatalizador del 4-NF con el propósito de aumentar el área superficial, logrando un porcentaje de degradación del $4-N F$ del $100 \%$ y un $88 \%$ de mineralización en 24 horas de irradiación. También se realizó la degradación del 4-NF usando dióxido de titanio dopado con oro (Au) alcanzándose un $100 \%$ de degradación del 4-NF (Naya et al., 2013).

Xin et al. (2014) sintetizaron nanotubos de titanio dopados con WO3, logrando la degradación del 98\% de 4-NF con un porcentaje de mineralización del $64 \%$, lo que evidencia que estas metodologías pueden ser susceptibles de ser aplicadas, no obstante, la electrodeposición puede traer consigo lodos activos que pueden tener mayor peligrosidad que los compuestos de partida.

En la tabla 4 se muestra los resultados de la degradación de 4-NF por diferentes investigadores, es importante destacar que hay metodologías comparativas, métodos más convencionales como la adsorción, bioadsorción y uso de membrana, con excelentes resultados, los procesos de oxidación avanzada siguen siendo un gran foco de interés debido a que representan un valor agregado y es que las especies oxidantes generadas en estas metodologías en general buscan la mineralización de los contaminantes de partida, y aunque con esto no se afirma que todos llegan a este término, forman subproductos de degradación que permiten la aplicación de metodologías en II etapas tales como los métodos biológicos, lo que sigue a continuación es investigar acerca de otros procesos de oxidación tal como el oxígeno molecular singulete, el cual no requiere la incorporación de reactivos tóxicos al tratamiento y aprovechando el oxígeno presente y la luz es capaz de degradar esta tipología de contaminantes, los cuales están siendo explorados como parte de sus procesos investigativos por los grupos de investigación involucrados en esta revisión.

Los métodos biológicos como lo planteado por (Cabana et al., 2007) usando un hongo de pudrición blanca Coriolopsis polizona, logró la degradación del 4-NF alrededor del 80\%. También se ha realizado la biodegradación en condiciones anaerobias del 4-NF (Chang et al., 2005; Lu et al., 2008) También existen otros sistemas más complejos como sistemas electrostáticos de alto voltaje para inmovilizar las células bacterianas o enzimas en microcápsulas de alginato para la eliminación de NF a partir de lodos de aguas residuales, aplicando un aumento de potencial de 0 a 12 $\mathrm{kV}$ generando remociones hasta de un $54.3 \%$ (Hsu et al.,2013) 
Tabla 4. Métodos de remoción consultados para el 4-nonilfenol

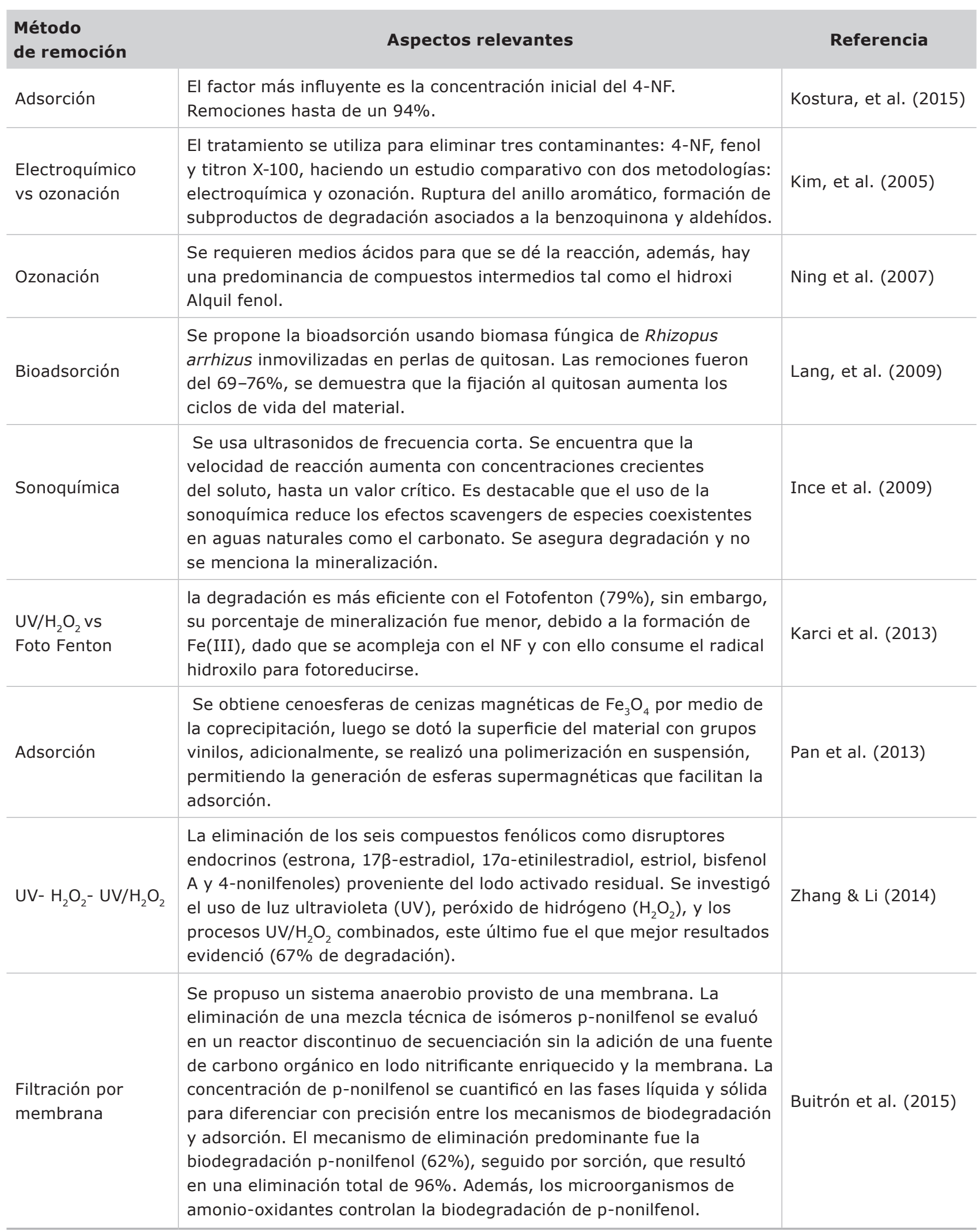




\section{CONCLUSIONES}

Los contaminantes emergentes como los alquilfenoles y sus isómeros, han sido detectados tanto en cuerpos de agua como en los sedimentos y todos los estudios resaltan su continuo aumento desde los años 90 como consecuencia de efectos tanto en animales como seres humanos, por lo que es de gran interés a nivel de salud pública por el incremento en el riesgo ambiental debido a su toxicidad especialmente a nivel endocrino. Por lo anterior, existe la búsqueda de metodologías de detección, cuantificación, remoción y degradación desdelos cuerpos de aguas y sedimentos. La cromatografía liquida de alta eficacia HPLC es una metodología ampliamente empleada en el análisis de los isómeros de los alquilfenoles, debido a su versatilidad. Dada la gran cantidad de isómeros generados, la cromatografía es la técnica mas utilizada para la cuantificación de nonilfenoles y sus isómeros, tal como se explicó en la serie de tiempos propuesta en el artículo.

Adicionalmente, también se destacan en tratamiento y degradación de estos contaminantes, el uso de los procesos de oxidación avanzada, que incluyen especies activas del oxígeno como el radical hidroxilo, que ha demostrado poca selectividad y gran eficacia en términos de degradación de estos compuestos, trayendo consigo la mineralización de varios de estos isómeros, por lo que los procesos de oxidación avanzada por su poca selectividad y capacidad de mineralización siguen siento una metodología efectiva de tratamiento para este tipo de contaminantes.

\section{AGRADECIMIENTOS}

A la Universidad Nacional de Colombia por la financiación a través del proyecto Hermes 35753 (QUIPU 203010019056).
A la Fundación para la promoción de la investigación y la tecnología a través del proyecto 3973 por la financiación de la investigación.

A COLCIENCIAS a través del programa de doctorados nacionales convocatoria 647 del 2015.

\section{LITERATURA CITADA}

Bechambi, O., Najjar, W., \& Sayadi, S. (2016). The nonylphenol degradation under UV irradiation in the presence of Ag-ZnO nanorods: Effect of parameters and degradation pathway. Journal of the Taiwan Institute of Chemical Engineers, (60), p.496-501.

Bokern, M., \& Harms, H. H. (1997). Toxicity and metabolism of 4-n-nonylphenol in cell suspension cultures of different plant species. Environmental Science and Technology, 31(7), p.1849-1854.

Bolong, N., Ismail, A. F., Salim, M. R., \& Matsuura, T. (2009). A review of the effects of emerging contaminants in wastewater and options for their removal. Desalination, 238(1-3), p. 229-246.

Buitrón, G., Torres-Bojorges, A. X., \& Cea-Barcia, G. (2015). Removal of p-nonylphenol isomers using nitrifying sludge in a membrane sequencing batch reactor. Chemical Engineering Journal, (281), p. 860-868.

Cabana, H., Jiwan, J. L. H., Rozenberg, R., ElisashviIi, V., Penninckx, M., Agathos, S. N., \& Jones, J. P. (2007). Elimination of endocrine disrupting chemicals nonylphenol and bisphenol $A$ and personal care product ingredient triclosan using enzyme preparation from the white rot fungus Coriolopsis polyzona. Chemosphere, 67(4), p.770-778.

Careghini, A., Mastorgio, A. F., Saponaro, S., \& Sezenna, E. (2014). Bisphenol A, nonylphenols, benzophenones, and benzotriazoles in soils, groundwater, surface water, sediments, and food: a review. Environmental Science and Pollution Research, (22), p. 5711-5741.

Céspedes, R., Skryjová, K., Raková, M., Zeravik, J., Fránek, M., Lacorte, S., \& Barceló, D. (2006). Validation of an enzyme-linked immunosorbent assay (ELISA) for the determination of 4-nonylphenol and octylphenol in surface water samples by LC-ESI-MS. Talanta, 70(4), p. 745-751. 
Chang, B. V., Chiang, F., \& Yuan, S. Y. (2005). Anaerobic degradation of nonylphenol in sludge. Chemosphere, 59(10), p.1415-1420.

Chen, R., Yin, P., Zhao, L., Yu, Q., Hong, A., \& Duan, S. (2014). Spatial-temporal distribution and potential ecological risk assessment of nonylphenol and octylphenol in riverine outlets of Pearl River Delta, China. Journal of Environmental Sciences (China), 26(11), p. 2340-2347.

Cheng, G., Sun, M., Yao, L., Wang, L., Sorial, G. A., Xu, X., \& Lou, L. (2015). Investigation into the feasibility of black carbon for remediation of nonylphenol polluted sediment through desorption kinetics after different order spiking. Chemosphere, (138), p. 568-575.

Cherniaev, A. P., Kondakova, A. S., \& Zyk, E. N. (2016). Contents of 4-Nonylphenol in Surface Sea Water of Amur Bay (Japan/East Sea). Achievements in the Life Sciences 10 (2016) p.65-71.

Deborde, M., Rabouan, S., Mazellier, P., Duguet, J. P., \& Legube, B. (2008). Oxidation of bisphenol A by ozone in aqueous solution. Water Research, 42(16), p.4299-4308.

Diehl, J., Johnson, S. E., Xia, K., West, A., \& Tomanek, L. (2012). The distribution of 4-nonylphenol in marine organisms of North American Pacific Coast estuaries. Chemosphere, 87(5), p.490-497.

Diehl, J., Johnson, S. E., Xia, K., West, A., Tomanek, L., Cortazar, E., Shi, W. (2005). Optimization of microwave-assisted extraction for the determination of nonylphenols and phthalate esters in sediment samples and comparison with pressurised solvent extraction. Chemosphere, 223(23), p.5616-5623.

Duan, P., Hu, C., Butler, H. J., Quan, C., Chen, W., Huang, W., Yang, K. (2016). Effects of 4-nonylphenol on spermatogenesis and induction of testicular apoptosis through oxidative stress-related pathways, (62), p.27-38.

Dulov, A., Dulova, N., \& Trapido, M. (2013). Photochemical degradation of nonylphenol in aqueous solution: The impact of $\mathrm{pH}$ and hydroxyl radical promoters. Journal of Environmental Sciences (China), 25(7), p.1326-1330.

Dzinun, H., Othman, M. H. D., Ismail, A. F., Puteh, M. H., Rahman, M. A., \& Jaafar, J. (2016). Photocatalytic degradation of nonylphenol using co-extruded dual-layer hollow fibre membranes incorporated with a different ratio of TiO2/PVDF. Reactive and Functional Polymers, (99), p.80-87.

EPA (2015). Use of High Throughput Assays and Computational Tools in the Endocrine Disruptor Screening Program. Recuperado de https:// www.epa.gov/endocrine-disruption/use-highthroughput-assays-and-computational-tools-endocrine-disruptor\#screening.

Fairbairn, D. J., Karpuzcu, M. E., Arnold, W. A., Barber, B. L., Kaufenberg, E. F., Koskinen, W. C., Swackhamer, D. L. (2016). Sources and transport of contaminants of emerging concern: $A$ two-year study of occurrence and spatiotemporal variation in a mixed land use watershed. Science of the Total Environment (551-552) p.605-613.

Gil, J., Soto, M., Usma, J., \& Gutiérrez, O. (2012). Contaminantes emergentes en aguas, efectos y posibles tratamientos. Producción Más Limpia, $7(2)$, p. 52-73.

Guenther, K., Heinke, V., Thiele, B., Kleist, E., Prast, H., \& Raecker, T. (2002). Endocrine disrupting nonylphenols are ubiquitous in food. Environ. Sci. Technol (36), p.1676-1680.

Guerreiro, A., Soares, A., Piletska, E., Mattiasson, B., \& Piletsky, S. (2008). Preliminary evaluation of new polymer matrix for solid-phase extraction of nonylphenol from water samples. Analytica Chimica Acta, 612(1), p.99-104.

Hsu, F. Y., Wang, Z. Y., \& Chang, B. V. (2013). Use of microcapsules with electrostatically immobilized bacterial cells or enzyme extract to remove nonylphenol in wastewater sludge. Chemosphere, 91(6), p.745-750.

Ince, N. H., Gültekin, I., \& Tezcanli-Güyer, G. (2009). Sonochemical destruction of nonylphenol: Effects of $\mathrm{pH}$ and hydroxyl radical scavengers. Journal of Hazardous Materials, 172(2-3), p.739-743.

Karci, A., Arslan-Alaton, I., \& Bekbolet, M. (2013). Advanced oxidation of a commercially important nonionic surfactant: Investigation of degradation products and toxicity. Journal of Hazardous Materials, (263), p.275-282.

Kim, J., Korshin, G. V., \& Velichenko, A. B. (2005). Comparative study of electrochemical degradation and ozonation of nonylphenol. Water Research, 39(12), p.2527-2534. 
Kostura, B., Škuta, R., Plachá, D., Kukutschová, J., \& Matýsek, D. (2015). Mg-Al-CO3 hydrotalcite removal of persistent organic disruptor - Nonylphenol from aqueous solutions. Applied Clay Science, (114), p.234-238.

Kuch, H. M., \& Ballschmiter, K. (2001). Determination of endocrine-disrupting phenolic compounds and estrogens in surface and drinking water by HRGC-(NCI)-MS in the picogram per liter range. Environmental Science and Technology, 35(15), p.3201-3206.

Kuzikova, I., Safronova, V., Zaytseva, T., \& Medvedeva, N. (2016). Fate and effects of nonylphenol in the filamentous fungus Penicillium expan- sum isolated from the bottom sediments of the Gulf of Finland. Journal of Marine Systems, in press.

Lang, W., Dejma, C., Sirisansaneeyakul, S., \& Sakairi, N. (2009). Biosorption of nonylphenol on dead biomass of Rhizopus arrhizus encapsulated in chitosan beads. Bioresource Technology, 100(23), p.5616-5623.

Lee, C. C., Jiang, L. Y., Kuo, Y. L., Hsieh, C. Y., Chen, C. S., \& Tien, C. J. (2013). The potential role of water quality parameters on occurrence of nonylphenol and bisphenol $A$ and identification of their discharge sources in the river ecosystems. Chemosphere, 91(7), p.904-911.

Lee, C., Jiang, L., Kuo, Y., Chen, C., Hsieh, C., Hung, C., \& Tien, C. (2015). Characteristics of nonylphenol and bisphenol an accumulation by fish and implications for ecological and human health, (502), p. 417-425.

Li, X., Chen, S., Li, L., Quan, X., \& Zhao, H. (2014). Electrochemically enhanced adsorption of nonylphenol on carbon nanotubes: Kinetics and isotherms study. Journal of Colloid and Interface Science, (415), p.159-164.

Lin, T., Yu, S., \& Chen, W. (2016). Occurrence, remo$\mathrm{val}$ and risk assessment of pharmaceutical and personal care products (PPCPS) in an advanced drinking water treatment plant (ADWTP) around Taihu Lake in China. Chemosphere, (152), p.1-9.

Loos, R., Hanke, G., Umlauf, G., \& Eisenreich, S. J. (2007). LC-MS-MS analysis and occurrence of octyl- and nonylphenol, their ethoxylates and their carboxylates in Belgian and Italian textile industry, wastewater treatment plant effluents and surface waters. Chemosphere, 66(4), p. 690-699.

Lu, J., Jin, Q., He, Y., Wu, J., \& Zhao, J. (2008). Biodegradation of nonylphenol polyethoxylates under sulfate-reducing conditions. Science of the Total Environment, 399(1-3), p.121-127.

Lu, Z., \& Gan, J. (2014). Analysis, toxicity, occurrence and biodegradation of nonylphenol isomers: A review. Environment International, (73), p.334-345.

Martinez-Zapata, M., Aristizábal, C., \& Peñuela, G. (2013). Photodegradation of the endocrine-disrupting chemicals $4 n$-nonylphenol and triclosan by simulated solar UV irradiation in aqueous solutions with $\mathrm{Fe}$ (III) and in the absence/presence of humic acids. Journal of Photochemistry and Photobiology A: Chemistry, (251), p.41-49.

Meador, J. P., Yeh, A., Young, G., \& Gallagher, E. P. (2016). Contaminants of emerging concern in a large temperate estuary. Environmental Pollution, (213), p.254-267.

Nakada, N., Tanishima, T., Shinohara, H., Kiri, K., \& Takada, H. (2006). Pharmaceutical chemicals and endocrine disrupters in municipal wastewater in Tokyo and their removal during activated sludge treatment. Water Research, 40(17), p.3297-3303.

Naya, S. I., Nikawa, T., Kimura, K., \& Tada, H. (2013). Rapid and complete removal of nonylphenol by gold nanoparticle/rutile titanium (IV) oxide plasmon photocatalyst. ACS Catalysis, 3(5), p.903-907.

Ning, B., Graham, N. J. D., \& Zhang, Y. (2007). Degradation of octylphenol and nonylphenol by ozone - Part II: Indirect reaction. Chemosphere, 68(6), p.1173-1179.

Noguera-Oviedo, K., \& Aga, D. S. (2016). Lessons Learned from more than Two Decades of Research on Emerging Contaminants in the Environment. Journal of Hazardous Materials (316) p. 242-251.

Pan, J., Li, L., Hang, H., Ou, H., Zhang, L., Yan, Y., \& Shi, W. (2013). Study on the nonylphenol removal from aqueous solution using magnetic molecularly imprinted polymers based on flyash-cenospheres. Chemical Engineering Journal, (223), p.824-832.

Peng, F., Ji, W., Zhu, F., Peng, D., Yang, M., Liu, R., ... Yin, L. (2016). A study on phthalate metabolites, bisphenol $A$ and nonylphenol in the urine of Chinese women with unexplained recurrent spontaneous abortion. Environmental Research, in press. 
Peng, J., Wang, G., Zhang, D., Zhang, D., \& Li, X. (2016). Journal of Photochemistry and Photobiology A: Chemistry Photodegradation of nonylphenol in aqueous solution by simulated solar UV-irradiation: The comprehensive effect of nitrate, ferric ion and bicarbonate, (326), p.9-15.

Pothitou, P., \& Voutsa, D. (2008). Endocrine disrupting compounds in municipal and industrial wastewater treatment plants in Northern Greece. Chemosphere, 73(11), p.1716-1723.

Rabouan, S., Dupuis, A., Cariot, A., Albouy-Llaty, M., Migeot, V., Cariot, A.; Legube, B. (2012). Analytical chemistry and metrological issues related to nonylphenols in environmental health. TrAC - Trends in Analytical Chemistry, (37), p.112-123.

Rodgers-gray, T. P., Jobling, S., Morris, S., Kelly, C., Kirby, S., Janbakhsh, A., Tyler, C. R. (2001). Long-term temporal changes in the estrogenic composition of treated sewage ef uent and its biological effects on fish. Environ. Sci. Technol., 34(8), p.1521-1528.

Ros, O., Vallejo, A., Blanco-Zubiaguirre, L., Olivares, M., Delgado, A., Etxebarria, N., \& Prieto, A. (2015). Microextraction with polyethersulfone for Bisphenol-A, alkylphenols and hormones determination in water samples by means of gas chromatography-mass spectrometry and liquid chromatography-tandem mass spectrometry analysis. Talanta, (134), p. 247-255.

Sayed, A. E. D. H., Mohamed, N. H., Ismail, M. A., Abdel-Mageed, W. M., \& Shoreit, A. A. M. (2016). Antioxidant and antiapoptotic activities of Calotropis procera latex on Catfish (Clarias gariepinus) exposed to toxic 4-nonylphenol. Ecotoxicology and Environmental Safety, (128), p.189-194.

She, Y., Wang, J., Zheng, Y., Cao, W., Wang, R., Dong, F., Wu, L. (2012). Determination of nonylphenol ethoxylate metabolites in vegetables and crops by high performance liquid chromatography-tandem mass spectrometry. Food Chemistry, 132(1), p.502-507.

Shirdel, I., \& Kalbassi, M. R. (2016). Effects of nonylphenol on key hormonal balances and histopathology of the endangered Caspian brown trout (Salmo trutta caspius). Comparative Biochemistry and Physiology Part - C: Toxicology and Pharmacology, (183-184), p. 28-35.

Soares, A., Guieysse, B., Jefferson, B., Cartmell, E., \& Lester, J. N. (2008). Nonylphenol in the environment: A critical review on occurrence, fate, toxicity and treatment in wastewaters. Environment International, 34(7), p.1033-1049.

Sumpter, J. P., \& Jobling, S. (1995). Vitellogenesis as a biomarker for estrogenic contamination of the aquatic environment. Environmental Health Perspectives, 103(SUPPL. 7), p.173-178.

Uchiyama, T., Makino, M., Saito, H., Katase, T., \& Fujimoto, Y. (2008). Syntheses and estrogenic activity of 4-nonylphenol isomers. Chemosphere, 73(1 SUPPL.), p.60-65.

Verderame, M., Prisco, M., Andreuccetti, P., Aniello, F., \& Limatola, E. (2011). Experimentally nonylphenol-polluted diet induces the expression of silent genes VTG and ERa in the liver of male lizard Podarcis sicula. Environmental Pollution, 159(5), p.1101-1107.

Villegas, L. G. C., Mashhadi, N., Chen, M., Mukherjee, D., Taylor, K. E., \& Biswas, N. (2016). A Short Review of Techniques for Phenol Removal from Wastewater. Current Pollution Reports, (2)157-167.

Vincent, M. D., \& Sneddon, J. (2009). Nonylphenol: An overview and its determination in oysters and wastewaters and preliminary degradation results from laboratory experiments. Microchemical Journal, 92(1), p. 112-118.

Wang, P. W., Chen, M. L., Huang, L. W., Yang, W., Wu, K. Y., \& Huang, Y. F. (2015). Prenatal nonylphenol exposure, oxidative and nitrative stress, and birth outcomes: A cohort study in Taiwan. Environmental Pollution, (207), p.145-151.

Xin, Y., Gao, M., Wang, Y., \& Ma, D. (2014). Photoelectrocatalytic degradation of 4-nonylphenol in water with WO3/TiO2 nanotube array photoelectrodes. Chemical Engineering Journal, (242), p.162-169.

Xu, L. J., Chu, W., Lee, P. H., \& Wang, J. (2016). The mechanism study of efficient degradation of hydrophobic nonylphenol in solution by a chemical-free technology of sonophotolysis. Journal of Hazardous Materials, (308), p.386-393.

Ying, G. G., Kookana, R. S., \& Ru, Y. J. (2002). Occurrence and fate of hormone steroids in the environment. Environment International (28) p. 545- 551.

Ying, G.-G., Williams, B., \& Kookana, R. (2002). Environmental fate of alkylphenols and alkylphenol ethoxylates: a review. Environment International, 28(3), p.215-226. 
Zgoła-Grześkowiak, A., Grześkowiak, T., Rydlichowski, R., \& Łukaszewski, Z. (2009). Determination of nonylphenol and short-chained nonylphenol ethoxylates in drain water from an agricultural area. Chemosphere, 75(4), p.513-518.
Zhang, A., \& Li, Y. (2014). Removal of phenolic endocrine disrupting compounds from waste activated sludge using UV, H2O2, and UV/H2O2 oxidation processes: Effects of reaction conditions and sludge matrix. Science of the Total Environment.

\section{(c) (i) () ()}

\section{Licencia de Creative Commons}

